Planetary Systems in the Universe - Observation, Formation and Evolution

Proceedings IAU Symposium No. 202, (c)2004 IAU

Alan Penny, Pawel Artymowicz, Anne-Marie Lagrange, \& Sara Russell, eds.

\title{
Penetration of Interstellar Dust Aggregates Into Circumstellar Dust Disks
}

\author{
Hiroshi Kimura \\ Institut für Planetologie, Westfälische Wilhelms-Universität, \\ Wilhelm-Klemm-Straße 10, D-48149 Münster, Germany \\ Ingrid Mann \\ Space Science Department ESTEC SCI-SO, PO Bus 299, 220 AG \\ Noordwijk, The Netherlands
}

\begin{abstract}
Interstellar dust particles, which are supposedly aggregates, penetrate dust disks around stars because of the star's motion relative to the surrounding interstellar medium (ISM). We discuss the interrelation of the physical properties of local interstellar dust, the relative motion of the star and the surrounding ISM, and the evolution of the circumstellar disk.
\end{abstract}

\section{Introduction}

The motion of a star relative to its surrounding interstellar medium (ISM) causes a flux of interstellar dust into the circumstellar dust disk. Because the stellar radiation exerts a repelling force on the grains in the vicinity of the star, the interaction between the circumstellar dust disks and the local interstellar dust is limited beyond the distance of the closest approach to stars for interstellar dust. Artymowicz \& Clampin (1997) estimated the effects of the radiation pressure on the distance of the closest approach to stars using Mie theory, which is the exact solution of Maxwell's equations for a sphere. Dust grains in the ISM are most probably not spherically shaped but rather aggregate particles in consequence of coagulation of colliding smaller grains (Ossenkopf 1993). In situ measurements of interstellar grains in the solar system indicate that this is the case for dust in the local interstellar cloud (LIC) surrounding the Sun (Mann \& Kimura 2000). In this paper, we evaluate the effect of the radiation pressure for the system of Beta Pictoris ( $\beta$ Pic) based on the analytical solution for an aggregate and discuss the interaction of interstellar dust with a circumstellar disk.

\section{Computational Methods}

The closest approach distances are a function of the speed of the star relative to the surrounding ISM and the radiation pressure on the grains. For the $\beta$ Pic system, Artymowicz \& Clampin (1997) assumed the relative speed of $8.6 \mathrm{~km} / \mathrm{s}$, which we will use as our estimate. The particle properties, which determine the radiation pressure, are not uniquely specified from available models of interstellar dust. Let interstellar dust be described as an aggregate of spherical 

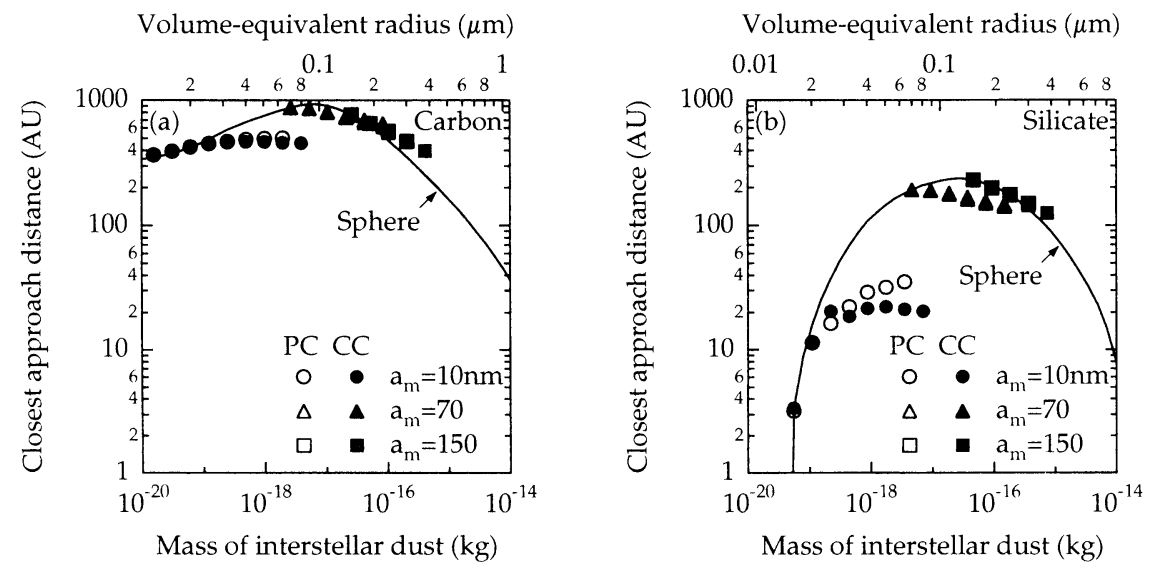

Figure 1. The closest approach distances for interstellar dust consisting of amorphous carbon (left) and silicate (right). The open and closed symbols indicate BPCAs and BCCAs, and the solid curve, spheres.

monomers identical in size and material composition grown by either ballistic particle-cluster aggregation (BPCA) or ballistic cluster-cluster aggregation (BCCA) processes (see Kimura \& Mann 1999). We consider $a_{\mathrm{m}}=10,70$, and $150 \mathrm{~nm}$ for the monomer radius, and amorphous carbon and silicate for the materials. The radiation pressure for the aggregates is calculated using the superposition $T$-matrix method, which effectively gives the analytical solution for a randomly oriented aggregate of spheres (Mackowski \& Mishchenko 1996).

\section{Results}

Figures $1 \mathrm{a}$ and $1 \mathrm{~b}$ show the closest approach distances for amorphous carbon and silicate grains. Aggregates in the mass range of $m \approx 10^{-19}-10^{-17} \mathrm{~kg}$ approach the star closer than spherical grains. For larger aggregates, the closest approach distances to the star can be comparable to or even larger than those of spherical grains. The distances for the aggregates become independent of their size and structure, but depend on the size and material of the monomers. Aggregates consisting of small monomers penetrate the disk deeper than those of large monomers. Amorphous carbon aggregates with $m \approx 10^{-17} \mathrm{~kg}$ approach no more than 500-900 AU. Silicate aggregates in the same mass range can reach distances of 20-200 AU from the center of the star. In this case, our results clearly differ from the results by Artymowicz \& Clampin (1997).

\section{Discussion}

Let us first re-examine the motion of $\beta$ Pic with respect to the surrounding ISM before going to discuss the interaction of dust and a star in the Local Bubble, in which $\beta$ Pic and the Sun are embedded. Recent observations with Hipparcos not only provide better information on the heliocentric motion of $\beta$ Pic but also 
revise the Sun's velocity with respect to the local standard of rest (LSR) (see Dehnen \& Binney 1998). In the LSR frame, the velocities of cloudlets in the Local Bubble point away from the Scorpius-Centaurus Association while only the LIC is found along the line of sight toward $\beta$ Pic (Lallement et al. 1995). This may indicate that the local ISM surrounding $\beta$ Pic moves with the same velocity as the LIC, yielding an estimate for the initial speed of interstellar dust toward $\beta$ Pic to be $20.5 \mathrm{~km} / \mathrm{s}$.

Adopting this speed, instead of $8.6 \mathrm{~km} / \mathrm{s}$, interstellar dust consisting of amorphous carbon and silicate can approach the zone $\lesssim 150$ AU from the star. We expect that dust aggregates consisting of composite material or core-mantle monomers also reach similar distances because their ratio $\beta$ of radiation pressure to gravity is close to the value for amorphous carbon grains. Graphite grains often discussed as a candidate material for the $217.5 \mathrm{~nm}$ interstellar extinction profile are expelled by the radiation pressure at larger distances because their $\beta$-values are higher than that of amorphous carbon grains. The asymmetry in the $\beta$ Pic disk observed between 150 and $800 \mathrm{AU}$ projected radius may result from the collisional interaction between interstellar dust and the circumstellar disk (Krivova et al. 2000). It is, however, difficult to conclude whether or not this interaction accounts for the observed disk asymmetry without taking into account the geometry of the disk and the direction of disk rotation with respect to the interstellar flow. On the other hand, impacts of interstellar grains on planetesimals can be a source of dust in the outer circumstellar disk as the case in the solar system (Yamamoto \& Mukai 1998). However, the dust density in the ISM around $\beta$ Pic might be too low to contribute to the evolution of the disk if the $\beta$ Pic system is immersed in low-density hot gas as expected for the Local Bubble, instead of higher-density warm gas, which surrounds the heliosphere (see Breitschwerdt et al. 1996). This issue requires further investigation with a better knowledge of the local ISM surrounding the circumstellar dust disk.

Acknowledgments. We would like to thank Daniel Mackowski, Kirk Fuller, and Michael Mishchenko for providing the superposition $T$-matrix code. One of us (H.K.) is indebted to the SOC of the symposium for financial support.

\section{References}

Artymowicz, P. \& Clampin, M. 1997, ApJ, 490, 863

Breitschwerdt, D., Freyberg, M. J. \& Egger, R. 2000, A\&A, 361, 303

Dehnen, W. \& Binney, J. J. 1998, MNRAS, 298, 387

Kimura, H. \& Mann, I. 1999, Phys. Chem. Earth, 24, 563

Krivova, N. A., Krivov, A. V. \& Mann, I. 2000, ApJ, 539, 424

Lallement, R., Ferlet, R., Lagrange, A. M., Lemoine, M. \& Vidal-Madjar, A. 1995, A\&A, 304, 461

Mann, I. \& Kimura, H. 2000, JGR, 357, 10317

Mackowski, D. W. \& Mishchenko, M. I. 1996, JOSA A, 13, 2266

Ossenkopf, V. 1993, A\&A, 280, 617

Yamamoto, S. \& Mukai, T. 1998, A\&A, 329, 785 